\title{
Um Estudo Exploratório sobre Plataformas Digitais para Ecossistemas de Inovação Social no Brasil
}

\author{
Luciana Chueri, Anderson Tavares, Mariana Pinheiro, Mateus Ramos, \\ Rodrigo Pereira dos Santos
}

\author{
Universidade Federal do Estado do Rio de Janeiro (UNIRIO) \\ Avenida Pasteur, 458 - Urca - CEP: 22290-255 - Rio de Janeiro, RJ, Brasil \\ \{luciana.chueri, anderson.afonso, mariana.pinheiro, mateus.ramos, \\ rps\} @uniriotec.br
}

\begin{abstract}
Due to the growing need for allowing different social innovation project actors to work in a coordinated and collaborative manner, the use of digital platforms has been considered as a great opportunity for social innovation ecosystems. However, in addition to technical issues, the existing challenges in the design and management of such platforms are combined with economic and social issues. This paper presents an exploratory study on real social innovation platforms to investigate functionalities that support these ecosystems. Transparency, process adaptability and coordination mechanisms were not observed. This study calls researchers/professionals to better design and manage such platforms in the context of social innovation.
\end{abstract}

Resumo. Devido à crescente necessidade de possibilitar que diferentes atores trabalhem de modo coordenado e colaborativo, o uso de plataformas digitais tem sido considerado uma oportunidade para ecossistemas de inovação social. Porém, além de questões técnicas, os desafios existentes no projeto e gestão dessas plataformas envolvem questões econômicas e sociais. Este artigo apresenta um estudo exploratório sobre plataformas reais de inovação social a fim de investigar funcionalidades que apoiam tais ecossistemas. Transparência, adaptabilidade de processos e mecanismos de coordenação não foram observados. Este estudo convida pesquisadores/profissionais a projetarem e gerenciarem melhor plataformas no contexto de inovação social.

\section{Introdução}

Atualmente, existe um consenso crescente entre profissionais, formuladores de políticas e comunidades de pesquisa de que somente inovações tecnológicas não são capazes de superar os desafios sociais e econômicos que as sociedades modernas estão enfrentando [Terstriep et al. 2020]. Nesse sentido, a importância de inovações sociais em abordarem de forma bem-sucedida os desafios sociais, econômicos, políticos e ambientais tem sido reconhecida globalmente [Domanski et al. 2019]. Sinteticamente, uma inovação social consiste de uma nova solução que atende a uma necessidade social de forma mais eficiente (se comparada às existentes), traz capacidades novas/melhoradas e viabiliza melhor uso de recursos na sociedade [Caulier-Grice et al. 2012].

Há numerosas e diversas iniciativas de inovação social sendo desenvolvidas de forma isolada [Howaldt et al. 2016], onde cada iniciativa trilha o seu próprio caminho e cada gestor faz a sua própria interpretação de conceitos e processos. Isoladamente, uma 
iniciativa pode até resultar em uma inovação social, mas de forma mais difícil e sem conexão ou colaboração com outros atores [Chueri et al. 2019]. Neste contexto, surge a necessidade de atores formarem redes e atuarem colaborativamente a fim de ampliar o desenvolvimento e a disseminação de inovações sociais [Howaldt et al. 2016; Domanski \& Kaletka 2018]. Um estudo publicado observou a necessidade de serviços associados a uma plataforma digital como suporte tecnológico a um ecossistema formado por atores envolvidos no desenvolvimento de inovações sociais [Chueri et al. 2019].

Estes ambientes onde são desenvolvidas inovações sociais são caracterizados pela participação de pessoas de diferentes setores econômicos e que atuam em diferentes organizações [Domanski et al. 2019], mas que desejam trabalhar colaborativamente em prol de um objetivo comum. A fim de estabelecer caminhos para realizar o suporte tecnológico a estes ambientes, conhecidos como ecossistemas de inovações sociais, é necessário identificar quais funcionalidade já são encontradas e quais necessitam ainda ser desenvolvidas. Nesse contexto, investigar fatores técnicos, humanos e organizacionais destas plataformas se torna necessário para melhorar a concepção, gerenciamento e evolução das soluções de apoio a este ecossistema.

Pelo fato de "ecossistemas de inovação social" ser um campo com estudos escassos [Braithwaite 2018], buscou-se o uso de instrumentos relacionados à investigação destes fatores na área de ecossistemas de software (ECOS), por apresentar estudos desta natureza. Dessa forma, o objetivo deste artigo é apresentar um estudo exploratório sobre plataformas reais de inovação social existentes no Brasil, utilizando os fatores de ECOS identificados por Santos et al. (2016). Espera-se que a análise de fatores técnicos, humanos e organizacionais possa ajudar pesquisadores e profissionais a projetarem e gerenciarem melhor essas plataformas no contexto de inovação social.

Este artigo está estruturado da seguinte forma: a Seção 2 apresenta a fundamentação teórica; a Seção 3 detalha a metodologia e a condução do estudo exploratório; a Seção 4 reporta a análise dos resultados obtidos; a Seção 5 apresenta as limitações e estratégias de mitigação utilizadas; por fim, a Seção 6 conclui o artigo com as considerações finais e perspectivas futuras a partir deste trabalho.

\section{Fundamentação Teórica}

\subsection{Ecossistemas de Inovação Social}

Inovações sociais são novas soluções (produtos, serviços etc.) que simultaneamente atendem a uma necessidade social de forma mais eficiente que soluções existentes, trazem capacidades novas ou melhoradas (e relacionamentos) e também viabilizam um melhor uso de recursos, aprimorando a capacidade de atuação da sociedade [CaulierGrice et al. 2012]. Estas inovações diferem da inovação tecnológica pelo processo que elas demandam (cooperativo e colaborativo) e pelo propósito (atender a necessidades sociais e criar novos relacionamentos e empoderamento dos participantes) [Howaldt et al. 2016]. Com o desenvolvimento crescente de iniciativas de inovação social, pesquisadores e profissionais estão movendo o foco de suporte a iniciativas isoladas para investigar formas de apoio a ambientes com inovações múltiplas e interconectadas.

Para Domanski \& Kaletka (2018), um ecossistema de inovação social consiste de atores que atuam em diferentes setores da sociedade e de seus ambientes com distintas normas legais e culturais, infraestruturas de suporte, entre outros elementos. 
Segundo Manzini (2015), esses ecossistemas são cruciais para promover, apoiar e desenvolver iniciativas bem-sucedidas de inovação social, assim como para criar as redes que permitem aos atores se envolverem e compartilharem ideias e inovação. Logo, é importante criar uma infraestrutura de apoio a estes atores, representada por uma plataforma bem estruturada para apoiar e conectar as diferentes iniciativas [Manzini 2015]. Neste artigo, é usada a definição de plataforma digital proposta por Tilson et al. (2012), que é composta por elementos técnicos (de software e hardware), processos organizacionais associados e padrões.

Estudos relacionados a ecossistemas de inovação social são recentes e escassos [Braithwaite 2018; Domanski et al. 2019], sobretudo quando direcionados à criação e análise de plataformas de apoio. Portanto, buscou-se em outros domínios formas de investigar plataformas por meio de dimensões que englobam fatores técnicos (i.e., tipos de infraestruturas digitais), humanos (i.e., relações entre pessoas e engajamento) e organizacionais (i.e., melhoria de processos e transparência).

\subsection{Ecossistemas de Software}

Existem diversos conceitos para caracterização de um ecossistema de software (ECOS) como, por exemplo, a interação entre software e atores a partir de uma plataforma tecnológica comum, resultando em um conjunto de contribuições que influenciam direta ou indiretamente o ecossistema [Manikas 2016]. Santos et al. (2016) definem ECOS como uma forma eficaz de construir software a partir de uma plataforma tecnológica comum, compondo aplicações e tecnologias desenvolvidas por múltiplos atores. Devido a essas características, identificou-se a possibilidade de aplicar instrumentos relacionados à visão de ECOS na investigação de plataformas de inovação social.

Pesquisas focam na identificação de como o conjunto de elementos que forma um ECOS tem gerado impacto nos diversos processos de gestão, que, por sua vez, depende de fatores técnicos e organizacionais influenciados por fatores humanos [Jansen et al. 2013]. Nesse sentido, Santos et al. (2016) identificaram uma lista de 15 fatores técnicos, humanos e organizacionais intrínsecos ao desenvolvimento de software no contexto de ECOS: (F1) Diversidade de organizações e relações de um ECOS; (F2) Estímulo de desenvolvedores externos a utilizarem uma plataforma central; (F3) Compartilhamento de conteúdo, conhecimento, problemas, experiências e habilidades, (F4) Melhoria na reutilização de software no cenário da Engenharia de Software globalizada, (F5) Reposicionamento das organizações para agirem como atores em rede e reduzirem a sua força de trabalho interna; (F6) Diversidade das novas funcionalidades fornecidas aos clientes; (F7) Transparência; (F8) Projeto modular de sistema; (F9) Abertura da organização; (F10) Definição de características internas relacionadas à saúde e à estabilidade do ECOS; (F11) Escopo/fronteiras do ECOS bem definidas; (F12) Identificação de capacidades e relacionamentos entre atores do ECOS; (F13) Definição clara de processos; (F14) Fortalecimento do caráter comunicativo inerente à programação; e (F15) Observância às características dos domínios de aplicação.

\section{Estudo Exploratório}

O estudo exploratório realizado seguiu a sequência de atividades ilustrada na Figura 1. Para a condução do estudo, foram convidados quatro especialistas com experiência no tema, com atuação na academia e na indústria, que são pesquisadores do laboratório 
envolvido há pelo menos um ano. Esta seção descreve os passos 1 a 6, sendo a análise qualitativa realizada na Seção 4 por meio da interpretação dos resultados do consenso.

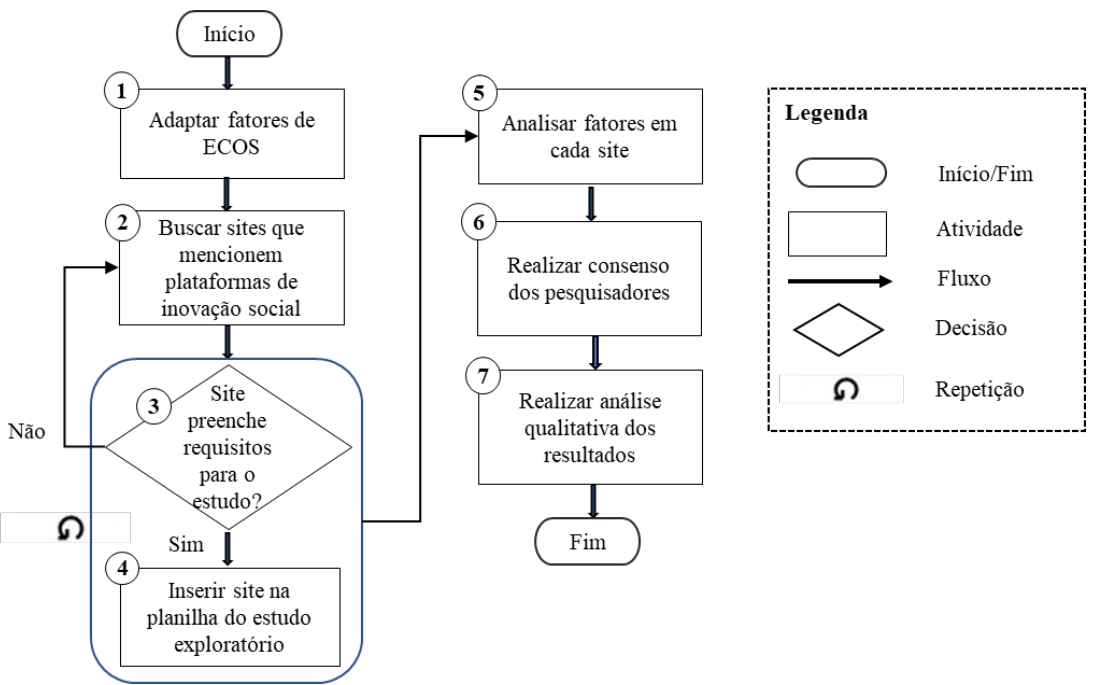

Figura 1. Procedimento do estudo exploratório.

A atividade de adaptação dos fatores de ECOS foi necessária para que pudessem ser investigados em ecossistemas de inovação social, uma vez que alguns fatores são relacionados a uma organização de desenvolvimento de software. Alguns fatores são específicos para ambientes de desenvolvimento de software e foram excluídos da análise (F6, F8 e F10). O conjunto de fatores utilizado no estudo e as adaptações feitas em alguns deles é apresentado na Tabela 1, destacando, em itálico, aqueles modificados.

Tabela 1. Adaptação dos fatores de ECOS para o contexto de inovação social.

\begin{tabular}{|c|c|c|}
\hline Fator & Fatores Originais (Santos et al. 2016) & Adaptação para o contexto de Inovação Social \\
\hline $\mathrm{F} 1$ & Diversidade de organizações e relações de um ECOS & Diversidade de atores e suas relações \\
\hline $\mathrm{F} 2$ & $\begin{array}{l}\text { Estímulo de desenvolvedores externos a utilizarem uma } \\
\text { plataforma central }\end{array}$ & Estimulo de atores a se engajarem em uma plataforma comum \\
\hline F3 & $\begin{array}{l}\text { Compartilhamento de conteúdo, conhecimento, problemas, } \\
\text { experiências e habilidades }\end{array}$ & $\begin{array}{l}\text { Compartilhamento de conteúdo, conhecimento, problemas, } \\
\text { experiências e habilidades }\end{array}$ \\
\hline F4 & $\begin{array}{l}\text { Melhoria na reutilização de software no cenário da } \\
\text { Engenharia de Software globalizada }\end{array}$ & $\begin{array}{l}\text { Melhoria na reutilização de inovações sociais em outras que } \\
\text { estão em estágio de desenvolvimento }\end{array}$ \\
\hline F5 & $\begin{array}{l}\text { Reposicionamento das organizações para agirem como } \\
\text { atores em rede e reduzirem a sua força de trabalho interna }\end{array}$ & Atores desejam se engajar para atuarem em rede \\
\hline F7 & Transparência & Transparência \\
\hline F9 & Abertura da organização & Abertura do ambiente e inovação aberta \\
\hline F11 & Escopo/fronteiras do ECOS bem definidas & Ambiente com fronteira bem definida \\
\hline F12 & $\begin{array}{l}\text { Identificação de capacidades e relacionamentos entre } \\
\text { atores do ECOS }\end{array}$ & $\begin{array}{l}\text { Mecanismos de coordenação e de reconhecimento de } \\
\text { capacidades }\end{array}$ \\
\hline F13 & Definição clara de processos & Processos adaptáveis \\
\hline F14 & $\begin{array}{l}\text { Fortalecimento do caráter comunicativo inerente à } \\
\text { programação }\end{array}$ & $\begin{array}{l}\text { Novos atores externos se unem a atores tradicionais } \\
\text { envolvidos no desenvolvimento de inovações sociais }\end{array}$ \\
\hline F15 & Observância às características dos domínios de aplicação & $\begin{array}{l}\text { A comunidade mantém o ambiente provendo demandas } e \\
\text { soluções para a plataforma }\end{array}$ \\
\hline
\end{tabular}

A motivação da escolha dos casos reais para conduzir o estudo exploratório se deve principalmente ao fato de se tratar de sites que mencionam a utilização de uma plataforma de inovação social, ou seja, ambientes com efetivas diretrizes de interação com a sociedade. As plataformas reais investigadas neste estudo foram encontradas a 
partir da execução de uma string na ferramenta de busca Google, realizada em setembro de 2019. Como o intuito foi investigar plataformas de inovação social brasileiras, foi utilizado o filtro para o idioma Português e foi excluída uma plataforma de um site português. A string de busca usada foi "plataforma de inovação social", com variações de acordo com o plural das palavras. A busca retornou uma lista de sites, apresentada na Tabela 2, onde cada site foi marcado com um identificador iniciado por $S$ a fim de facilitar sua utilização nas planilhas do estudo.

Tabela 2. Seleção preliminar de casos reais para o estudo.

\begin{tabular}{|c|l|c|l|}
\hline Id. & Sites retornados na busca & Inclui? & \multicolumn{1}{|c|}{ Justificativa } \\
\hline S1 & Aoka $^{1}$ & Não & Consultoria para negócios \\
\hline S2 & Novos Urbanos $^{2}$ & Não & Site sobre uma iniciativa social \\
\hline S3 & Inturma $^{3}$ & Não & Site indisponível \\
\hline S4 & Rede Filantropia $^{4}$ & Não & Plataforma de treinamentos pagos \\
\hline S5 & Gife $^{5}$ & Não & Associação de empresas do terceiro setor \\
\hline S6 & PrecisaSER $^{6}$ & Não & Site indisponível \\
\hline S7 & Instituto Nexxera $^{7}$ & Não & Site indisponível \\
\hline S8 & Lab de Inovação em Finanças Sociais $^{8}$ & Sim & \\
\hline S9 & Observatório de Inovação Social de Florianópolis $^{9}$ & Sim & \\
\hline S10 & Inovasocial $^{10}$ & Não & Plataforma para disseminação de conteúdos \\
\hline S11 & Umov.me Arena $^{11}$ & Não & Site para desenvolvimento e suporte de aplicativos \\
\hline S12 & Social Good Brasil $^{12}$ & Sim & \\
\hline
\end{tabular}

Os sites que retornaram na busca foram analisados para verificar se eles representavam um ambiente de apoio a atores de inovações sociais. Os seguintes critérios de exclusão foram aplicados a cada site: i) site está indisponível (casos S3, S6 e $\mathrm{S} 7$ ); ii) site representa um negócio oferecendo consultoria e/ou treinamento (casos S1, S4 e S11); iii) site representa um portal informativo de uma associação de empresas (caso S5); e iv) site representa uma única iniciativa social (caso S2). Como em cada critério de inclusão, foi observado se o site mencionava diversos atores e se representava algum ambiente de apoio a eles. Desta forma, os casos S8, S9 e S12 foram selecionados para a próxima atividade do estudo.

Os sites selecionados foram: i) S8- Laboratório de Inovação em Finanças Sociais: Laboratório que reúne lideranças de diferentes organizações (empresas, institutos, incubadoras, governo etc.) para identificar oportunidades no campo e cocriar protótipos de ação colaborativa; ii) S9- Observatório de Inovação Social de Florianópolis: mapeia os atores de suporte e iniciativas de inovação social do Ecossistema de Inovação Social de Florianópolis; e iii) S12- Social Good Brasil:

\footnotetext{
${ }^{1} \mathrm{http}: / /$ www.aoka.com.br

${ }^{2}$ http://www.novosurbanos.com.br

${ }^{3} \mathrm{http}: / /$ www.inturma.com.br

${ }^{4} \mathrm{http}: / / \mathrm{www}$.filantropia.ong

${ }^{5} \mathrm{http}: / /$ www.gife.com.br

${ }^{6} \mathrm{http}: / /$ www.precisaser.com.br

${ }^{7} \mathrm{http}: / /$ www.institutonexxera.org.br

${ }^{8} \mathrm{http}: / /$ www.labinovacaofinanceira.com

${ }^{9} \mathrm{http}: / /$ www.observafloripa.com.br

${ }^{10} \mathrm{http} / / / \mathrm{www}$.inovasocial.com.br

${ }^{11} \mathrm{http}: / /$ www.umov.me

$12 \mathrm{http}: / /$ www.socialgoodbrasil.org.br
} 
parceira da Fundação das Nações Unidas, Parceira da Fundação das Nações Unidas, que incentiva ao uso de tecnologias, dados e competências do futuro para o bem comum.

Cada um dos quatro especialistas recebeu uma planilha contendo os links dos três sites e a planilha de fatores adaptados a fim de investigar, para cada site, quais fatores estavam contemplados, parcialmente contemplados ou não contemplados. Em seguida, os resultados foram tabulados e foi realizada uma reunião de consenso entre os especialistas. $\mathrm{O}$ resultado desta atividade é apresentado na Tabela 3.

Tabela 3. Fatores observados nas plataformas.

\begin{tabular}{|c|c|c|c|c|c|c|}
\hline Site & Fator & Especialista 1 & Especialista 2 & Especialista 3 & Especialista 4 & Consenso \\
\hline \multirow{12}{*}{ S8 } & F1 & Sim & Sim & Sim & Sim & Sim \\
\hline & $\mathrm{F} 2$ & Não & Não & Não & Não & Não \\
\hline & F3 & Sim & Sim & Sim & Sim & Sim \\
\hline & $\mathrm{F} 4$ & Não & Não & Não & Não & Não \\
\hline & F5 & Sim & Não & Parcial & Parcial & Parcial \\
\hline & F7 & Não & Parcial & Não & Não & Não \\
\hline & F9 & Sim & Não & Sim & Sim & Sim \\
\hline & F11 & Não & Não & Não & Não & Não \\
\hline & F12 & Sim & Não & Não & Não & Não \\
\hline & F13 & Não & Não & Sim & Não & Não \\
\hline & F14 & Sim & Sim & Sim & Sim & Sim \\
\hline & F15 & Não & Não & Não & Não & Não \\
\hline \multirow{12}{*}{ S9 } & $\mathrm{F} 1$ & Sim & Parcial & $\mathrm{Sim}$ & Sim & Sim \\
\hline & $\mathrm{F} 2$ & Sim & Sim & Sim & Sim & Sim \\
\hline & F3 & Sim & Sim & Sim & Sim & Sim \\
\hline & F4 & Não & Não & Não & Não & Não \\
\hline & F5 & Sim & $\mathrm{Sim}$ & Parcial & Sim & Sim \\
\hline & F7 & Não & Não & Sim & Não & Não \\
\hline & F9 & Sim & Sim & Sim & Sim & Sim \\
\hline & F11 & Não & Sim & Não & Não & Não \\
\hline & F12 & Não & Não & Não & Não & Não \\
\hline & F13 & Não & Não & Sim & Sim & Não \\
\hline & F14 & Sim & Sim & Sim & Sim & Sim \\
\hline & F15 & Não & $\operatorname{Sim}$ & Parcial & Parcial & Parcial \\
\hline \multirow{12}{*}{ S12 } & F1 & Sim & Sim & Sim & Sim & Sim \\
\hline & $\mathrm{F} 2$ & Não & Não & Não & Não & Não \\
\hline & F3 & Sim & Sim & Parcial & Sim & Sim \\
\hline & F4 & Não & Não & Não & Não & Não \\
\hline & F5 & Não & Sim & Não & Não & Não \\
\hline & F7 & Não & Não & Não & Não & Não \\
\hline & F9 & Sim & Sim & Não & Sim & Sim \\
\hline & F11 & Não & Sim & Não & Não & Não \\
\hline & F12 & Não & Não & Não & Não & Não \\
\hline & F13 & Não & Parcial & Não & Não & Não \\
\hline & F14 & Sim & Sim & Não & Sim & Sim \\
\hline & F15 & Não & Não & Não & Não & Não \\
\hline
\end{tabular}

\section{Discussão dos Resultados}

A análise qualitativa realizada nesta seção remete à interpretação dos resultados decorrentes da reunião de consenso, face à literatura relacionada ao campo de inovação 
social. Os resultados obtidos neste estudo exploratório permitiram identificar que a perspectiva técnica dos ecossistemas de inovação social se encontra em fase de crescimento, dados que os sites são recentes e foram encontrados apenas três sites com plataformas de apoio aos atores. Este número pequeno (12 sites identificados inicialmente) pode estar relacionado à compreensão e ao uso do termo "plataforma", que pode ser entendido como um portal informativo ou um site para venda de serviços, entre outros.

Não foram observados os fatores F4, F7, F12 e F13 na etapa de consenso em nenhuma das três plataformas. Não foram identificadas informações relacionadas ao processo de desenvolvimento das plataformas, de documentos de projeto a código fonte ou tarefas de desenvolvimento [Santos et al. 2016], o que representaria o fator de transparência (F7). A ausência de F11 é justificada pelo fato dos três sites não apresentarem as fronteiras dos ambientes de inovação social. Com relação a F12, não foram identificados mecanismos de coordenação, embora estes possam existir internamente na plataforma, mas não estarem disponíveis a usuários que as consultam. A ausência de mecanismos de coordenação com apoio digital também foi observada em um estudo exploratório sobre um ecossistema de inovação social real [Chueri et al. 2019]. A adaptabilidade de processos (F13) e a reutilização (F4) não foram identificadas em nenhum dos três sites.

Foram observados os fatores F1, F3, F9, F14 nas análises. Percebeu-se que há uma compreensão nos papéis que cada ator exerce no ecossistema (F1) pela análise dos sites; inclusive, S9 apresenta formas de cadastro diferenciado para cada tipo de ator. Este dado corrobora estudos reportando diferentes papéis de atores de ecossistemas de inovação social, como Domanski \& Kaletka (2018) e Chueri et al. (2019). Com relação a F3, foi identificado compartilhamento de conteúdo de caráter informativo, descrição de experiências e casos de sucesso. Contudo, nenhum dos três sites compartilha informações sobre habilidades dos atores. A abertura (F9) foi identificada nos três sites e está alinhada ao conceito de abertura dos ecossistemas de inovação social que, segundo Santos et al. (2016), é um fator crítico e envolve aspectos de compartilhamento de conhecimento (F3). F14 está presente e alinhado a uma das características de ecossistemas de inovação social dado que, nestes ambientes, organizações do terceiro setor que atuam tradicionalmente com iniciativas sociais atuam em conjunto com organizações orientadas a lucro, entre outros [Chueri \& Araujo 2018].

Foram parcialmente observados os fatores F2, F5, F11 e F15. O estímulo ao uso de uma plataforma central (F2) foi mais fortemente evidenciado em S9, que possui uma seção do site onde as informações dos atores do ecossistema estão registradas. O desejo dos atores em se engajarem para atuar em rede (F5) é observado em dois dos 3 sites, por isto no consenso entre os 3 sites foi considerado que este resultado seria "parcial". No entanto, não foi observado o reposicionamento de atores internos para agirem como atores em rede, ou mesmo mecanismos para identificar relacionamentos entre atores. Nicolopoulou et al. (2017) apontam que, na inovação social, a colaboração é fator primordial e pode ser expandida por redes de relacionamento. Não foram identificadas funcionalidades de apoio para estas redes, corroborando a percepção de Drake (2018) de que o estudo de redes de colaboração no campo de inovação social é pouco evidenciado [Drake 2018]. F11 foi considerado parcialmente atendido pois o site S9 possui uma delimitação clara ao ser voltado para as inovações sociais da cidade de Florianópolis, ao passo que S8 e S12 não apresentaram nenhuma informação sobre fronteiras ou limites. 
Com relação ao fator F15, não foram observados nos sites formas dos atores fornecerem demandas e ou soluções para a plataforma.

Em uma pesquisa de opinião sobre colaboração em ambientes reais de inovação social publicada anteriormente foram identificadas pelos participantes 41 ferramentas de apoio [Pinheiro et al., 2020]. Contudo, na análise, não foi observada nenhuma comunicação ou menção a ferramentas desta natureza. $\mathrm{O}$ acesso a estas ferramentas por meio de links ou integrações poderia promover diversidade de novas funcionalidades fornecidas aos clientes (F6).

As descobertas do estudo exploratório reforçam os resultados de um mapeamento sistemático de literatura relacionado aos desafios encontrados por atores no desenvolvimento de projetos de inovação social. O mapeamento apontou, como alguns dos maiores desafios, a presença de raras soluções tecnológicas de apoio a estes atores alinhado à carência de processos, além da falta de habilidades e competências para desenvolver os projetos [Chueri \& Araujo 2018]. Estes resultados estão alinhados à ausência ou pouca percepção na identificação de fatores associados a estes itens nas plataformas observadas. As percepções discutidas, resultantes do mapeamento sistemático, do estudo exploratório realizado por Chueri et al. (2019) e ainda deste estudo exploratório, expõem a realidade das plataformas de apoio aos ecossistemas de inovação social no Brasil. Adicionalmente, tais percepções enfatizam a necessidade de evolução das soluções tecnológicas de suporte aos atores destes ecossistemas.

A respeito de trabalhos relacionados, não foram encontrados estudos com o mesmo objetivo do presente estudo. Os 15 fatores identificados por Santos et al. (2016) foram utilizados por Fernandes \& Santos (2017) em um estudo exploratório em um ecossistema de software real. O objetivo do estudo foi identificar quais fatores são percebidos e possuem influência direta na interoperabilidade do ECOS SIGA, um sistema corporativo de grande porte em ambiente web - contexto diferente deste estudo.

\section{Limitações}

Como limitações do estudo, podemos citar: i) utilização de fatores de outro domínio de ecossistema (no caso, de software); e ii) a análise de fatores por parte dos especialistas teve um caráter subjetivo resultando na diferença de opiniões.

Para mitigar impactos que possam ter ocorrido devido às limitações relatadas, foram adotadas as seguintes estratégias: i) o caráter subjetivo das análises foi reduzido pela participação de quatro especialistas e a realização da reunião de consenso; e ii) a ameaça decorrente do caráter subjetivo das análises é uma particularidade dos estudos qualitativos e foi reduzida com a participação de um pesquisador sênior, que revisou os dados e as análises do estudo.

\section{Considerações Finais}

O objetivo deste artigo foi apresentar um estudo exploratório sobre a percepção de fatores técnicos, humanos e organizacionais em plataformas reais de inovação social, que dão apoio a seus ecossistemas. Para isso, foram adaptados os fatores de ECOS para o contexto de ecossistemas de inovação social, mais especificamente no que se refere a plataformas tecnológicas de apoio. Após a identificação de sites relacionados a estas plataformas, apenas três foram considerados como suporte a ambientes de inovação social. Estes sites foram analisados por quatro especialistas na área. 
O consenso percebido pelos especialistas no contexto das plataformas investigadas indicou que quatro fatores foram observados (F1, F3, F9, F14), quatro foram parcialmente observados (F2, F5, F11 e F15) e quatro não foram observados (F4, F7, F12 e F13). Este resultado, alinhado à pouca quantidade de plataformas identificadas, reflete a novidade do assunto sobre plataformas de suporte a inovações sociais no Brasil.

O estudo realizado também permitiu identificar a situação da perspectiva técnica dos ecossistemas de inovação social no Brasil, que pode se apresentar de duas formas: i) sem uma plataforma digital de apoio, conforme relatado em um estudo exploratório descrito em Chueri et al. (2019); e ii) com plataformas de apoio ainda em fase inicial, se comparadas a plataformas de outros ecossistemas, como ECOS. Esta percepção está alinhada à realidade do campo de estudo de ecossistemas de inovações sociais, que se mostra recente e pouco desenvolvido [Domanski et al. 2019].

A partir deste trabalho, espera-se estimular pesquisadores e profissionais a terem uma visão abrangente de fatores técnicos, humanos e organizacionais em plataformas de apoio a ecossistemas de inovação social. Alguns trabalhos futuros e oportunidades foram identificados a partir deste estudo, tais como: i) aplicar os passos deste estudo em sites de plataformas internacionais, de forma a obter uma amostra maior para análise; ii) investigar requisitos de qualidade presentes nas plataformas; e iii) investigar quais são os mecanismos de coordenação utilizados pelos gestores das plataformas. Além disso, no contexto de sistemas de informação, os gaps apresentados pelo não tratamento de alguns fatores abrem caminhos para a investigação de soluções tecnológicas específicas de apoio aos atores de inovação social.

\section{Agradecimentos}

O presente trabalho foi realizado com apoio da Coordenação de Aperfeiçoamento de Pessoal de Nível Superior - Brasil (CAPES) - Código de Financiamento 001, do Conselho Nacional de Desenvolvimento Científico e Tecnológico (CNPq) e da Universidade Federal do Estado do Rio de Janeiro (UNIRIO) - projeto n ${ }^{\circ}$ X0002/2019.

\section{Referências}

Braithwaite, P. (2018) Building Change Trust: Social Innovation Ecosystems: Building Change Trust, Belfast.

Butzin, A., Terstriep, J. (2018) Actor and roles in social innovation. In: Howaldt, J.; Kaletka, C.; Schröder, A.; Zirngiebl, M. (Eds), Atlas of Social Innovation - New Practices for a Better Future, pp. 77-81. TU Dortmund University: Dortmund.

Caulier-Grice, J., Davies, A., Patrick, R., Norman, W. (2012) Defining social innovation. TEPSIE, European Commission - 7th Framework Programme, Brussels: European Commission, DG Research.

Chueri, L.O.V., Araujo, R. M. (2018) How social innovation projects are managed? Answers from a literature review. European Public \& Social Innovation Review $3(2): 23-36$.

Chueri, L.O.V., Vasconcelos, A.P.V., Santos. R.P. (2019) An observational study on the challenges faced by actors in a social innovation ecosystem. In: Proceedings of 11 th 
International Conference on Management of Digital EcoSystems (MEDES'19), Limassol, Chipre, pp. 219-223.

Domanski, D., Kaletka, C. (2018) Social Innovation Ecosystems. In: Howaldt, J.; Kaletka, C.; Schröder, A.; Zirngiebl, M. (Eds), Atlas of Social Innovation, New Practices for a Better Future, pp.207-211. TU Dortmund University: Dortmund.

Domanski, D., Howaldt, J., Kaletka, C. (2019) A comprehensive concept of social innovation and its implications for the local context - on the growing importance of social innovation ecosystems and infrastructures. European Planning Studies, 28(3):454-474, DOI: 10.1080/09654313.2019.1639397.

Drake, I. (2018) Social Innovation and Collaboration. Identifying and Engaging Stakeholders with Power, Purpose, Passion and Presence. In: E. Torgersen (Ed) Interaction: Samhandling Under Risk. pp. 213-232. Oslo: Cappelen Damm.

Fernandes, J., Santos, R.P. (2017) Estudo Exploratório sobre Interoperabilidade no Ecossistema SIGA: Uma Análise das Dimensões do ePING. In: VIII Workshop sobre Aspectos da Interação Humano-Computador Web Social, Joinville, Brasil, pp 13-24.

Howaldt, J., Kaletka, C., Schröder, A., Terstriep, J., Christoph, R., Dieter, T. (2016) Mapping the World of Social Innovation: A Global Comparative Analysis across Sectors and World Regions. Technical Report, Technische Universität Dortmund.

Jansen, S., Brinkkemper, S., Cusumano, M. (2013) Software Ecosystems: Analyzing and Managing Business Networks in the Software Industry. 1ed., Cheltenham/UK, Northampton/USA: Edward Elgar Publishing.

Manzini, E. (2015) Design, When Everybody Designs: An Introduction to Design for Social Innovation. Cambridge, Massachusetts: The MIT Press.

Manikas, K., (2016) Revisiting software ecosystems research. The Journal of Systems and Software 117(2016):84-103.

Nicolopoulou, K., Karataş-Özkan, M., Vas, C., Nouman, M. (2017) An incubation perspective on social innovation: the London Hub - a social incubator. $R \& D$ Management 47(3):368-384.

Pinheiro, M. C., Chueri, L.O.V., Santos, R.P. (2020) Identifying Topics and Difficulties on Collaboration in Social Innovation Environments. In: XVI Brazilian Symposium on Information Systems (SBSI’20), São Bernardo do Campo, Brasil.

Santos, R. P., Viana, D., Maciel, C. (2016) Ecossistemas de Software: Uma Visão sobre Fatores Técnicos, Humanos e Organizacionais". In: Gasparini, I.; Mota, M. (Org.). Livro dos Tutoriais do XV IHC. 15ed. Porto Alegre: SBC, v. C, pp. 70-90.

Terstriep, J., Rehfeld, D., Kleverbeck, M. (2020) Favourable social innovation ecosystem(s)? - An explorative approach. European Planning Studies 28:5, 881-905.

Tilson, D., Sørensen, C., Lyytinen, K. (2012) Change and Control Paradoxes in Mobile Infrastructure Innovation: The Android and iOS Mobile Operating Systems Cases, In: Proceedings of the 45th Hawaii International Conference on System Sciences, Maui, HI, 2012, pp. 1324-1333. 